\title{
High viral load suggests increased COVID-19 severity in a longitudinal cohort
}

\section{Yuanlin He}

Nanjing Medical University Department of Epidemiology

$\mathrm{Xin} \mathrm{Xu}$

Nanjing Medical University Department of Epidemiology

\section{Qun Lu}

Nanjing Medical University Department of Epidemiology

Zhiliang Hu

Nanjing University of Chinese Medicine the Second Hospital of Nanjing

Yue Jiang

Nanjing Medical University Department of Epidemiology

Ci Song

Nanjing Medical University Department of Epidemiology

\section{Wei Chen}

Nanjing University of Chinese Medicine the Second Hospital of Nanjing

\section{Peipei Li}

Suzhou Institute of Systems Medicine

\section{Weixiao Wang}

Nanjing University of Chinese Medicine the Second Hospital of Nanjing

\section{Chuanjun Xu}

Changzhou Women and Children's Hospital

\section{Yuting Ma}

Suzhou Institute of Systems Medicine

\section{Yuan Lin}

Nanjing Medicial University Department of Maternal

\section{Zhibin Hu}

Nanjing Medical University Department of Epidemiology

\section{Yongxiang Yi}

Nanjing University of Chinese Medicine the Second Hospital of Nanjing Hongbing Shen ( $\nabla$ hbshen@njmu.edu.cn )

Nanjing Medical University 
Keywords: 2019 novel coronavirus, viral load, co-infection, HPBV

Posted Date: June 12th, 2020

DOI: https://doi.org/10.21203/rs.3.rs-34267/v1

License: (c) (i) This work is licensed under a Creative Commons Attribution 4.0 International License. Read Full License 


\section{High viral load suggests increased COVID-19 severity in a longitudinal cohort}

Yuanlin $\mathrm{He}^{1,2^{*}}$, Xin $\mathrm{Xu}^{1,2^{*}}$, Qun $\mathrm{Lu}^{1,2^{*}}$, Zhiliang $\mathrm{Hu}^{3^{*}}$, Yue Jiang ${ }^{1,2}$, Ci Song ${ }^{1,2}$, Wei

Chen ${ }^{4}$, Peipei $\mathrm{Li}^{5}$, Weixiao Wang ${ }^{4}$, Chuanjun $\mathrm{Xu}^{6}$, Yuting Ma ${ }^{5}$, Yuan $\mathrm{Lin}^{7}$, Zhibin $\mathrm{Hu}^{1,2}$, Yongxiang $\mathrm{Yi}^{3 \dagger}$, Hongbing Shen ${ }^{1,2 \dagger}$

${ }^{1}$ Department of Epidemiology, Center for Global Health, School of Public Health, Nanjing Medical University, Nanjing 211166, China;

${ }^{2}$ State Key Laboratory of Reproductive Medicine, Nanjing Medical University, Nanjing 211166, China;

${ }^{3}$ Nanjing Infectious Disease Center, the Second Hospital of Nanjing, Nanjing University of Chinese Medicine, Nanjing 210003, China;

${ }^{4}$ Department of Clinical Research Center, the Second Hospital of Nanjing, Nanjing University of Chinese Medicine, Nanjing 210003, China;

${ }^{5}$ Suzhou Institute of Systems Medicine, Suzhou 215123, China;

${ }^{6}$ Department of Radiology, the Second Hospital of Nanjing, Nanjing University of Chinese Medicine, Nanjing 210003, China;

${ }^{7}$ Department of Maternal, Child and Adolescent Health, School of Public Health, Nanjing Medical University, Nanjing 211166, China

${ }^{*}$ These authors contributed equally to this work.

${ }^{\dagger}$ Correspondence to: Hongbing Shen, Department of Epidemiology, Center for Global Health, School of Public Health, Nanjing Medical University, 101 Longmian Road, Nanjing 211166, China. Tel: +86-25-86868437, Fax: +86-25-86868437. E-mail: hbshen@njmu.edu.cn; Yongxiang Yi, Nanjing Infectious Disease Center, the Second Hospital of Nanjing, Nanjing University of Chinese Medicine, 1-1 Zhongfu Road, Nanjing 210003, China, Tel: +025-83626203, E-mail: ian0126@126.com. 


\begin{abstract}
Background: An outbreak caused by the 2019 novel coronavirus (2019-nCoV) has spread globally. However, the viral dynamics, co-infection and their associations with clinical severity, have not been well explored.

Methods: We longitudinally enrolled 23 (Five severe-type, ten common-type and eight asymptomatic-type patients) hospitalized 2019-nCov-infected patients in Jiangsu between January 21 and February 11, 2020. Medical records and pharyngeal swab specimens, were collected to analyze the association between viral dynamic and disease severity.
\end{abstract}

Results: Five severe-type, ten common-type and eight asymptomatic-type patients were enrolled. Linear mixed effects models revealed that the common and severe-type patients had a higher level of viral load (3.08 points, 95\% CI, 0.51-5.65, $P=0.019 ; 6.07$ points, 95\% CI, 2.79-9.35, $P<0.001)$ and maintained a higher peak viral load $(P=$ 0.066 and 0.022 , respectively), when compared with the asymptomatic group. Viral load shedding among older patients (aged $\geq 60$ ) processed slower than that among younger patients $(P=0.047)$. RNA virome sequencing identified two co-infected RNA viruses, Human endogenous retrovirus H (HERV) and Human picobirnavirus (HPBV). Of note, HPBV was detected in one severe-type and two common-type patients, while was not detected in all the asymptomatic cases.

Conclusion: Higher viral load was positively associated with disease severity. This finding highlights the importance of monitoring the viral kinetics to identify patients at greater risk of progressing to severe pneumonia.

Keywords: 2019 novel coronavirus, viral load, co-infection, HPBV 


\section{Background}

The COVID-19 caused by 2019-nCoV infection represents a spectrum of clinical severity. Some patients are asymptomatic or have merely mild upper respiratory tract symptoms. The virus also causes severe pneumonia. It is estimated that approximately $20 \%$ of patients develop severe respiratory illness eventually, with the overall case fatality rate around $2.3 \%[1]$. Hence, it is critical to compare the clinical features of COVID-19 patients diagnosed with different clinical severity and to identify the risk factors for the progression to severe COVID-19 pneumonia.

Early efforts on describing the temporal profiles of viral load have been done for 2019-nCov[2-4]. A previous observational cohort study revealed that the viral load profile of 2019-nCoV peaks at symptom onset[4]. Other case series reported that 2019nCoV could shed in upper/lower respiratory specimens, stools, blood and urines of patients[5, 6]. However, important knowledge gaps remain, particularly regarding the temporal profiles of viral load in association with disease severity of COVID-19. On the other hand, several studies have reported the co-infection of 2019-nCov with other respiratory virus, like Influenza A[7]. The co-infection indicates the possibility of viral interactions bearing on the course of infectious diseases[8, 9]. However, the exact roles of co-infection in the clinical course of COVID-19 are still unclear.

Here we longitudinally assessed the clinical symptoms and severity of 23 hospitalized 2019-nCov-infected patients in Jiangsu, and systematically evaluated their viral load and mixed infection in relation to disease severity. 


\section{Methods}

\section{Study design and patient enrollment}

This study was approved by the Institutional Review Board (IRB) of Nanjing Medical University. A total of 23 COVID-19 patients were recruited in this study in Jiangsu Province. All patients were enrolled between January 21 and February 11, 2020 in this cohort study, with last follow-up was on March 14, 2020. All patients enrolled in this study were diagnosed as COVID-19 according to the diagnostic criteria from the seventh version of the guidelines on the Diagnosis and Treatment of COVID-19 by the National Health Commission of China[10]. Based on the guideline, patients were classified into three groups: severe type; asymptomatic type; and common type. Patient are classified as severe if one of the following conditions is met: 1) respiratory rate $\geq$ 30 per min; 2) oxygen saturation on room air at rest $\leq 93 \%$; or 3 ) partial pressure of oxygen in arterial blood/fraction of inspired oxygen $\leq 300 \mathrm{mmHg}$. Patients who are tested positive for 2019-nCoV virus' nucleic acid but have no clinical symptoms are defined as asymptomatic infection. The rest patients are classified as common type. In our study, severe type and common type were combined as symptomatic group (vs. asymptomatic group) in the analysis.

\section{Data source}

Medical records of COVID-19 patients were reviewed and contact history, demographic, clinical, laboratory examination, and outcome data were collected by the research team. The symptoms presented on each case during their hospitalization were recorded in this study, including fever, cough, nasal congestion, dizziness, fatigue, arthralgia, etc. Laboratory assessments included whole blood count, blood chemistry, coagulation test, liver and renal function, electrolytes, C-reactive protein, procalcitonin, lactate dehydrogenase and creatine kinase. Pharyngeal swab specimens were collected on admission day and every other day thereafter for the 2019-nCov virus test. Viral RNA was isolated from $200 \mu$ l virus preservation solution containing throat swabs using the AllPrep DNA/RNA Mini Kit (Qiagen, Hilden, Germany) following the manufacturer's instructions[11]. Quantitative real-time reverse-transcriptase 
polymerase chain reaction (qRT-PCR) for the Orflab gene was performed with qRTPCR kit (BGI Genomics, Beijing, China) recommended by the Chinese Center for Disease Control and Prevention (CDC) following WHO guidelines [10, 12]. The specimens were considered positive if the cycle threshold $\left(\mathrm{C}_{t}\right)$ value was $\leq 40$, and negative if the results were undetermined.

\section{Virus RNA library construction and sequencing}

For each patient, the sample on admission was selected for RNA virome sequencing. Virus RNA, as described above, was reverse transcribed, amplified using REPLI-g WTA Single Cell Kit (Qiagen, Hilden, Germany) and Ovation Trio RNA-Seq library preparation kit (NuGEN, CA, USA) and sequenced on the NovaSeq platform (Illumina). Library preparation and sequencing were carried out at Nanjing Medical University, Nanjing, China.

\section{Virome sequencing data processing}

The raw sequencing reads were first adaptor and quality trimmed using the Trimmomatic program at a quality filter of Q20 Phred quality score[13]. Host human genome reads were filted by removed using the STAR program[14]. The remaining reads were assembled de novo using Megahit[15] with default parameter settings. Megahit generated a total of 1,312,368 assembled contigs with a size range of 20022832 nt. The number of reads of each assembled contig was calculated using BBmap[16]. All of these assembled contigs were compared (using BLASTn and Diamond BLASTx) against the entire virus non-redundant (nr) protein sequence database and nucleotide (nt) sequence database (NCBI taxonomy ID 10239), with e values set to $1 \times 10^{-3}$, mismatch set to $\leq 3$ and match length set to $\geq 50$ and $\geq 100$, respectively. For contigs mapped to virus reference sequence, false-positive contigs were removed by comparing against the entire $\mathrm{nr}$ and nt databases (not only virus) with e values set to $1 \times 10^{-3}$.

\section{Statistical analysis}


Continuous variables were expressed as the medians and interquartile ranges (IQR). Categorical variables were summarized as the counts and percentages in each category. Fisher's exact test was used for categorical variables. Mann-Whitney U test was used to compare the $\mathrm{C}_{\mathrm{t}}$ value among different clinical groups. Spearman's correlation was applied for evaluating the relationship between age and $C_{t}$ value. To describe distinct clusters of individual trajectories, trajectory models were fit using the STATA 'traj' within each clinical group. Each trajectory could be estimated with up to a first-order polynomial function of the dependent variable, in this case $C_{t}$, over time. Linear mixed effects models were applicable for comparing the viral dynamics among different subgroups. All statistical analyses were performed using STATA (v15.0, College Station, TX, USA) and significance was determined at a $P<0.05$. 


\section{Results}

\section{Description of Clinical Characteristics}

The demographic and clinical characteristics were listed in Table S1. The median age was 41.0 years (IQR, 23.0-64.0). Ten patients (43.5\%) were males. Twelve (52.2\%) had a history of recent travel to Hubei. One patient had a history of smoking, and 7 (30.4\%) were complicated with chronic diseases. The most common symptoms at illness onset were fever $(15,65.2 \%)$ and cough $(9,39.1 \%)$.

According to the guidelines, 5 patients were classified into severe group (one was critical case), 8 were asymptomatic group, and the rest 10 patients with non-severe pneumonia onset wereclassified into common group. Compared with asymptomatic or common patients, the severe patients had a higher rate of diabetes (12.5\% vs. $0 \%$ vs. $60.0 \%, P=0.018)$, fever (0\% vs. $100.0 \%$ vs. $100.0 \%, P<0.001)$, cough $(0 \%$ vs. $50.0 \%$ vs. $80.0 \%, P=0.009)$, nausea $(0 \%$ vs. $0 \%$ vs. $60.0 \%, P=0.006)$ and vomit $(0 \%$ vs. $0 \%$ vs. $40.0 \%, P=0.040$ ). The laboratory findings at admission were also listed in Table S1. Patients with severe disease had more abnormal laboratory test results (including leukopenia and lymphocytopenia, $P=0.036$ and 0.040 , respectively) than those with non-severe disease. All of the 23 patients received antiviral treatment and interferon atomization, $11(47.8 \%)$ were given empirical antibiotic treatment. All 5 severe patients received protease inhibitor, antibiotic treatment, corticosteroids and immunoglobin. One $(20.0 \%)$ among them received antifungal treatment, and one was given oxygen support. Upon the complete of the study (March 14, 2020), all the patients were discharged, and no patients died.

\section{9-nCov RNA profiles}

In total, 260 pharyngeal swab specimens were obtained from 23 patients (mean 11.3 pharyngeal swab specimens per patient). Specimens with undetectable viral load were assigned a $C_{t}$ value of 40 cycle. No significant correlation was noted between age and baseline/peak $\mathrm{C}_{\mathrm{t}}$ value $(P=0.637,0.056$, respectively, Fig. 1A, 1B). No association was seen between baseline $C_{t}$ value and severity of illness $(P=0.897$ for asymptomatic vs. symptomatic; $P=0.768$ for asymptomatic vs. severe; Fig. 1C, 1E). Nevertheless, a 
significant positive association between peak viral load and disease severity was noted. Patients of symptomatic group and severe group maintained a higher peak viral load (showed as a lower $\mathrm{C}_{\mathrm{t}}$ value) when compared with asymptomatic patients $(P=0.017$ for asymptomatic vs. symptomatic; $P=0.022$ for asymptomatic vs. severe; Fig. 1D, 1F).

All the patients (100.0\%) had cleared 2019-nCov after a median 16.0 days (IQR 13.0-25.0 days) of follow-up. For each clinical group (age $<60$, age $\geq 60$; severe, common, asymptomatic group and symptomatic group), long-term $\mathrm{C}_{\mathrm{t}}$ value profile was fitted respectively and graphically summarized in Fig. 2. Linear mixed effects models were used to compare the between-group variance. As shown in Fig. 2, viral load $\left(C_{t}\right.$ value) of all the subgroups declined along with time ( $P$ for time $<0.001)$. The viral load of patients aged $\geq 60$ declined slower than that in patients aged $<60(P=0.047$, Fig. 2A). Compared with the asymptomatic group, the common and severe group had a higher level of viral load (3.08 points, 95\% CI, 0.51-5.65, $P=0.019 ; 6.07$ points, $95 \%$ CI, 2.79-9.35, $P<0.001$; Fig. 2C). The combined symptomatic group also maintained a higher level of viral load (3.82 points, 95\% CI, 1.32-6.33, $P=0.003$; Fig. 2B) when compared with the asymptomatic group. However, the interaction terms between disease severity and time did not show significant effects on viral load patterns $\left(C_{t}\right.$ value).

\section{Co-infection of human picobirnavirus (HPBV)}

Overall, 18 of 23 samples sequenced successfully (78.3\%), which includes 5 asymptomatic-type, 8 common-type and 5 severe-type patients. After RNA virome mapping, three RNA virome among all of the samples was identified (Fig. 3), including 2019-nCov, Human endogenous retrovirus H (HERV) and Human picobirnavirus (HPBV). 2019-nCov was detected among all of the patients. Of note, HPBV was detected in samples of one severe-type and two common-type patients, while was not detected in any asymptomatic case. 


\section{Discussion}

This study summarized the viral load and mixed infection of 23 hospitalized 2019nCov-infected patients in Jiangsu. 2019-nCov-infected patients were divided into severe group, common group and asymptomatic group according to the criteria set in the guidelines on the Diagnosis and Treatment of COVID-19 issued by the National Health Commission of China.

In our cohort, a high viral load on presentation of COVID-19 was observed even for asymptomatic carriers. The baseline viral load detected in the asymptomatic patient was similar to that in the symptomatic patients, which suggests that 2019-nCov can be transmitted easily, even for asymptomatic carriers. This finding could account for the efficient transmission from asymptomatic carriers and person-to-person transmission noted in community and health-care settings $[12,17]$. But, we observed that disease severity was associated with a higher peak viral load, which means that the viral load may be an independent factor for COVID-19 outcome. This finding suggests that viral load should be monitored and analyzed, so that it can be considered in the interpretation of outcome data.

We further prospectively characterized SARS-CoV-2 viral dynamics in the patient cohort. Our data provided important findings for this newly discovered virus infected in human. First, 2019-nCov in pharyngeal swab shedding within one month, the shedding period was consistent with former studies[18]. Second, 2019-nCov in patients with older age shed slower than that in younger age, which took account for the association between old age and worse prognosis in COVID-19. As interpreted in a previous SARS-CoV study[19], older age, as expected for immunosenescence, was an independent factor associated with higher viral load. Third, viral load levels in pharyngeal swab were correlated with clinical severity. The symptomatic patients maintained a higher level of viral load when compared with asymptomatic patients. Previous SARS researchers found that high plasma concentrations of SARS were related to the short-term adverse outcome of ICU admission and the ultimate outcome in terms of death[20,21]. The findings also suggest a weaker transmission capacity of asymptomatic cases due to the lower viral load. 
A previous study has detected the co-infection of 2019-nCov and influenza A virus one case in Wuhan[22]. Our study documented the co-infection of the 2019-nCov with HPBV among symptomatic patients. HPBV are small, non-enveloped viruses with a bipartite genome of double-stranded RNA. Studies in immunocompromised persons suggest that picobirnaviruses may be opportunistic enteric pathogens[23, 24]. A Previous study has determined the new picobirnaviruses in the respiratory tracts of patients with respiratory disease of unknown origin[25]. These observation expand our knowledge that picobirnaviruses may opportunistically replicate in the respiratory tracts of patients with 2019-nCov. However, further robust epidemiological evidence, histopathological and electron microscope observation is urgently needed, to better understand the existence of HPBV co-infection, and their interaction bearing on the disease severity.

Nevertheless, there are some limitations for our study. First, only cycle threshold values, not exact viral loads were collected in our study. Second, large-scale, multicenter cohorts from other regions are needed to verify our preliminary findings. In conclusion, COVID-19 is an emerging infection with many unknowns. This study has shed light on viral kinetics and mixed infection in COVID-19 patients and provided scientific evidence for guiding infection control policies and therapeutics. Further virological and functional studies are needed to reveal the underlying mechanism of severe COVID-19.

\section{Conclusions}

In short, our data suggested that higher viral load was positively associated with COVID-19 severity. This finding highlights the importance of monitoring the viral kinetics to identify patients at greater risk of progressing to severe pneumonia.

\section{Additional files}

Additional file 1: Supplementary tables (DOCX $23.2 \mathrm{~kb}$ ) 


\begin{abstract}
Abbreviations
qRT-PCR: Quantitative real-time reverse-transcriptase polymerase chain reaction; $\mathrm{C}_{\mathrm{t}}$ : cycle threshold; Nr: non-redundant protein sequence database; Nt: nucleotide sequence database; IQR: interquartile ranges; HERV: Human endogenous retrovirus H; HPBV: Human picobirnavirus
\end{abstract}

\title{
Acknowledgement
}

This study was funded in part by the National Natural Science Foundation of China (Special project, 82041026); the National Natural Science Foundation of China (81903382), the Natural Science Foundation of Jiangsu Province (BK20190652), China Postdoctoral Science Foundation (General Progaram, 2019M651900, 2019M651894). Written informed consent was obtained from each enrolled patient.

\section{Author contributions}

Hongbing Shen, Zhibin Hu and Yongxiang Yi made substantial contributions to the study concept and design. Zhiliang Hu took responsibility for the acquisition and check of data. Zhiliang $\mathrm{Hu}$ and Qun Lu took responsibility for analysis and interpretation of the data; Yuanlin He and Yue Jiang were responsible for virus RNA library construction; $\mathrm{Xin} \mathrm{Xu}$ was in charge of the manuscript draft. Xin $\mathrm{Xu}$ took responsibility for the statistical analysis; Ci Song, Wei Chen, Peipei Li, Weixiao Wang, Chuanjun Xu, Yuting Ma and Yuan Lin provided administrative, technical, and material support; Zhibin $\mathrm{Hu}$, Hongbing Shen and Yongxiang Yi made substantial revisions to the manuscript.

\section{Funding}

This study was supported by the National Natural Science Foundation of China(No.82041026, 81903382), the Natural Science Foundation of Jiangsu Province (BK20190652), China Postdoctoral Science Foundation (General Progaram, 2019M651900).

\section{Availability of data and materials}


Sequence reads generated in this study are available from the NCBI Sequence Read Archive (SRA) database under BioProject accession number PRJNA631802.

\section{Consent for publication}

Not applicable.

\section{Competing interests}

We declare no competing interests.

\section{Ethics approval and consent to participate}

This study was reviewed and approved by the Institutional Review Board (IRB) of Nanjing Medical University (approval number 2020-008). 


\section{Reference}

1. Team NCPERE: The epidemiological characteristics of an outbreak of 2019 novel coronavirus diseases (COVID-19) in China. Zhonghua Liu Xing Bing Xue Za Zhi 2020, 41:145-151.

2. Xu T, Chen C, Zhu Z, Cui M, Chen C, Dai H, Xue Y: Clinical features and dynamics of viral load in imported and non-imported patients with COVID-19. Int J Infect Dis 2020, 94:68-71.

3. Zou L, Ruan F, Huang M, Liang L, Huang H, Hong Z, Yu J, Kang M, Song Y, Xia J, et al: SARS-CoV-2 Viral Load in Upper Respiratory Specimens of Infected Patients. $N$ Eng/ J Med 2020, 382:1177-1179.

4. To KK, Tsang OT, Leung WS, Tam AR, Wu TC, Lung DC, Yip CC, Cai JP, Chan JM, Chik TS, et al: Temporal profiles of viral load in posterior oropharyngeal saliva samples and serum antibody responses during infection by SARS-CoV-2: an observational cohort study. Lancet Infect Dis 2020, 20:565-574.

5. Peng L, Liu J, Xu W, Luo Q, Deng K, Lin B, Gao Z: 2019 Novel Coronavirus can be detected in urine, blood, anal swabs and oropharyngeal swabs samples. medRxiv 2020:2020.2002.2021.20026179.

6. Wang W, Xu Y, Gao R, Lu R, Han K, Wu G, Tan W: Detection of SARS-CoV-2 in Different Types of Clinical Specimens. Jama 2020.

7. Khodamoradi Z, Moghadami M, Lotfi M: Co-infection of Coronavirus Disease 2019 and Influenza A: A Report from Iran. Arch Iran Med 2020, 23:239-243.

8. Pou AM, Rimell FL, Jordan JA, Shoemaker DL, Johnson JT, Barua P, Post JC, 
Ehrlich GD: Adult respiratory papillomatosis: human papillomavirus type and viral coinfections as predictors of prognosis. Ann Otol Rhinol Laryngo/ 1995, 104:758-762.

9. Franz A, Adams O, Willems R, Bonzel L, Neuhausen N, Schweizer-Krantz S, Ruggeberg JU, Willers R, Henrich B, Schroten H, Tenenbaum T: Correlation of viral load of respiratory pathogens and co-infections with disease severity in children hospitalized for lower respiratory tract infection. J Clin Viro/2010, 48:239-245.

10. China NHCotPsRo: New Coronavirus Pneumonia Prevention and Control Program (7th edn).

http://wwwnhcgovcn/yzygj/s7653p/202003/46c9294a7dfe4cef80dc7f5912eb1989shtm 12020.

11. Grabmüller M, Madea B, Courts C: Comparative evaluation of different extraction and quantification methods for forensic RNA analysis. Forensic Sci Int Genet 2015, 16:195-202.

12. Hu Z, Song C, Xu C, Jin G, Chen Y, Xu X, Ma H, Chen W, Lin Y, Zheng Y, et al: Clinical characteristics of 24 asymptomatic infections with COVID-19 screened among close contacts in Nanjing, China. Sci China Life Sci2020, 63:706-711.

13. Bolger AM, Lohse M, Usadel B: Trimmomatic: a flexible trimmer for Illumina sequence data. Bioinformatics 2014, 30:2114-2120.

14. Dobin A, Davis CA, Schlesinger F, Drenkow J, Zaleski C, Jha S, Batut P, Chaisson M, Gingeras TR: STAR: ultrafast universal RNA-seq aligner. Bioinformatics 2013, 29:15-21.

15. Li D, Liu CM, Luo R, Sadakane K, Lam TW: MEGAHIT: an ultra-fast single-node 
solution for large and complex metagenomics assembly via succinct de Bruijn graph. Bioinformatics 2015, 31:1674-1676.

16. Bushnell B: BBMap short-read aligner, and other bioinformatics tools. 2015.

17. Jiang XL, Zhang XL, Zhao XN, Li CB, Lei J, Kou ZQ, Sun WK, Hang Y, Gao F, Ji SX, et al: Transmission potential of asymptomatic and paucisymptomatic SARS-CoV-2 infections: a three-family cluster study in China. $J$ Infect Dis 2020.

18. Tan W, Lu Y, Zhang J, Wang J, Dan Y, Tan Z, He X, Qian C, Sun Q, Hu Q, et al: Viral Kinetics and Antibody Responses in Patients with COVID-19. medRxiv 2020:2020.2003.2024.20042382.

19. Chu CM, Poon LL, Cheng VC, Chan KS, Hung IF, Wong MM, Chan KH, Leung WS, Tang BS, Chan VL, et al: Initial viral load and the outcomes of SARS. Cmaj 2004, $171: 1349-1352$.

20. Ng EK, Hui DS, Chan KC, Hung EC, Chiu RW, Lee N, Wu A, Chim SS, Tong YK, Sung JJ, et al: Quantitative analysis and prognostic implication of SARS coronavirus RNA in the plasma and serum of patients with severe acute respiratory syndrome. Clin Chem 2003, 49:1976-1980.

21. Cheng VC, Hung IF, Tang BS, Chu CM, Wong MM, Chan KH, Wu AK, Tse DM, Chan $\mathrm{KS}$, Zheng BJ, et al: Viral replication in the nasopharynx is associated with diarrhea in patients with severe acute respiratory syndrome. Clin Infect Dis 2004, 38:467-475.

22. Wu X, Cai Y, Huang X, Yu X, Zhao L, Wang F, Li Q, Gu S, Xu T, Li Y, et al: Coinfection with SARS-CoV-2 and Influenza A Virus in Patient with Pneumonia, China. Emerg Infect Dis 2020, 26. 
23. Giordano MO, Martinez LC, Rinaldi D, Gúinard S, Naretto E, Casero R, Yacci MR, Depetris AR, Medeot SI, Nates SV: Detection of picobirnavirus in HIV-infected patients with diarrhea in Argentina. J Acquir Immune Defic Syndr Hum Retrovirol 1998, 18:380-383.

24. Martínez LC, Giordano MO, Isa MB, Alvarado LF, Paván JV, Rinaldi D, Nates SV: Molecular diversity of partial-length genomic segment 2 of human picobirnavirus. Intervirology 2003, 46:207-213.

25. Smits SL, van Leeuwen M, Schapendonk CM, Schürch AC, Bodewes R, Haagmans $\mathrm{BL}$, Osterhaus $\mathrm{AD}$ : Picobirnaviruses in the human respiratory tract. Emerg Infect Dis 2012, 18:1539-1540.

\section{Figure Legends}

Figure 1. Association between baseline/peak $C_{t}$ value and clinical characteristics. Correlation between age and initial viral load (A). Correlation between age and peak viral load (B). Comparison of initial (C) and peak (D) viral load between asymptomatic and symptomatic cases. Comparison of initial (E) and peak (F) viral load among asymptomatic, common and severe cases. 
Figure 2. 2019-nCov RNA shedding among different clinical groups. Linear mixed models were used to compare the between-group variance, baseline Age (A), Asymptomatic and symptomatic group (B), the common, severe and asymptomatic group $(\mathrm{C})$.

Figure 3. The proportion of three RNA virus through RNA virome sequencing. 
Figures

(A)

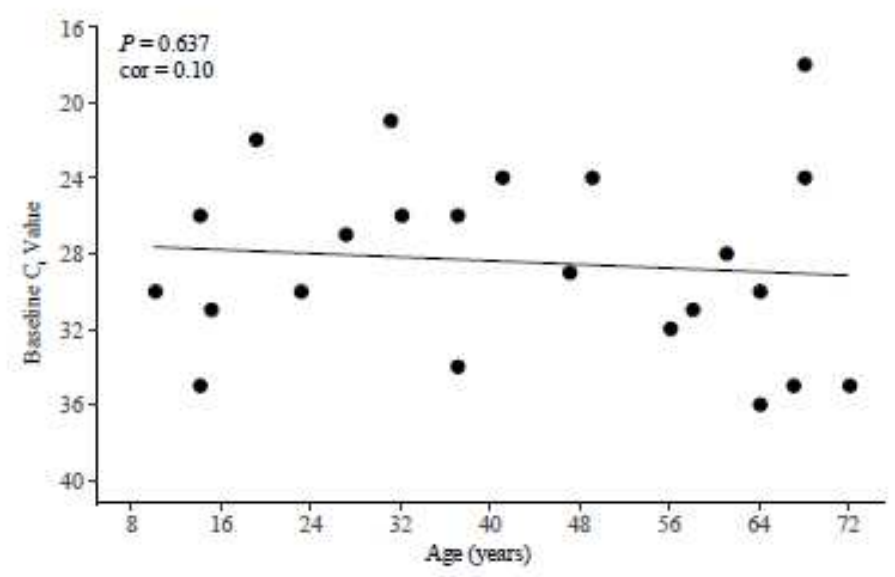

(C)

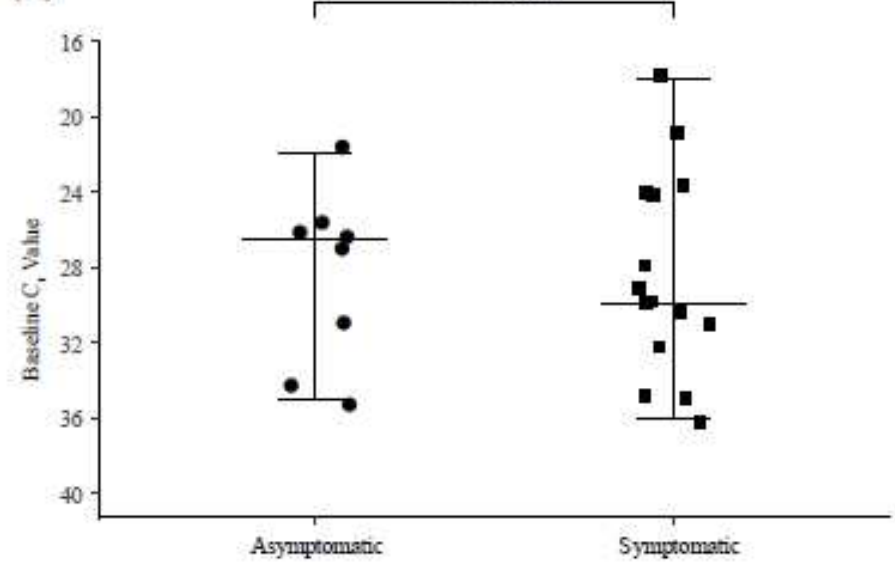

(E)

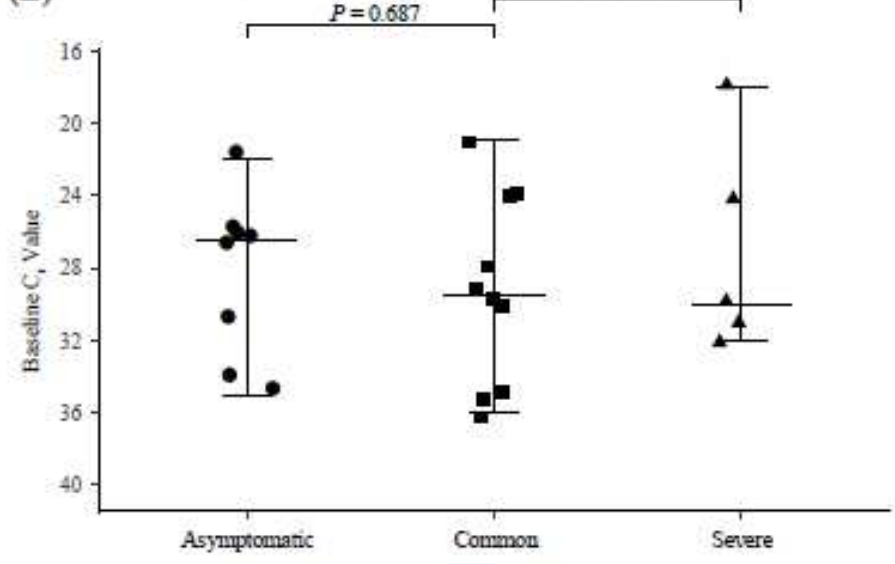

(B)

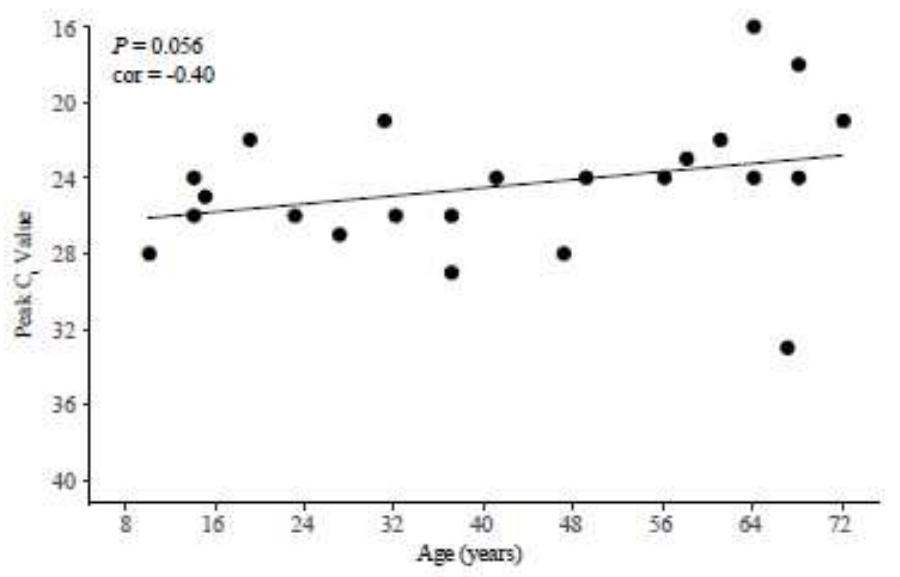

(D)

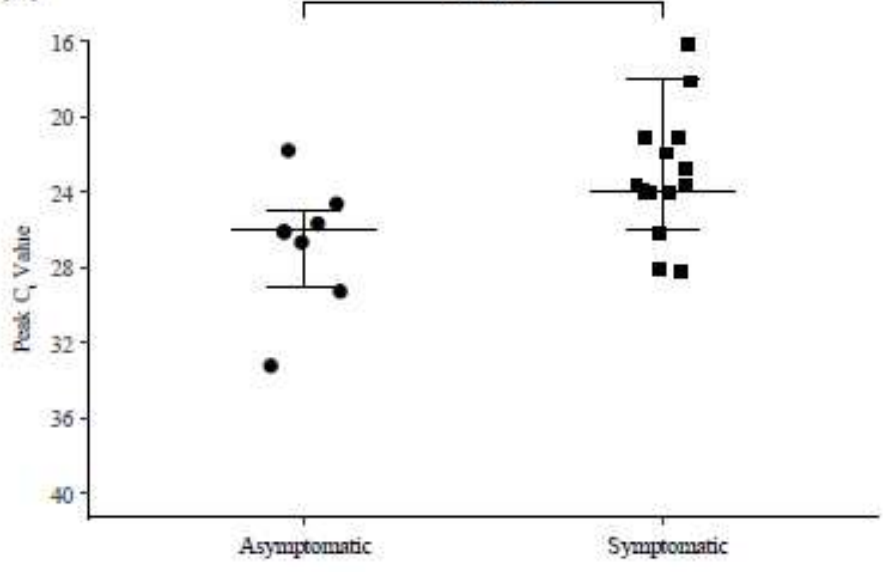

(F)

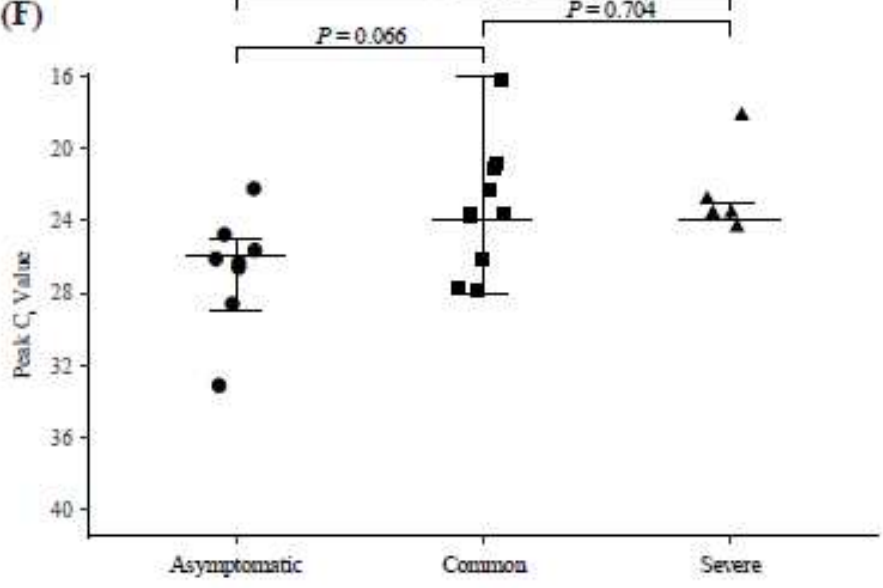

Figure 1

Association between baseline/peak Ct value and clinical characteristics. Correlation between age and initial viral load (A). Correlation between age and peak viral load (B). Comparison of initial (C) and peak 
(D) viral load between asymptomatic and symptomatic cases. Comparison of initial (E) and peak (F) viral load among asymptomatic, common and severe cases.

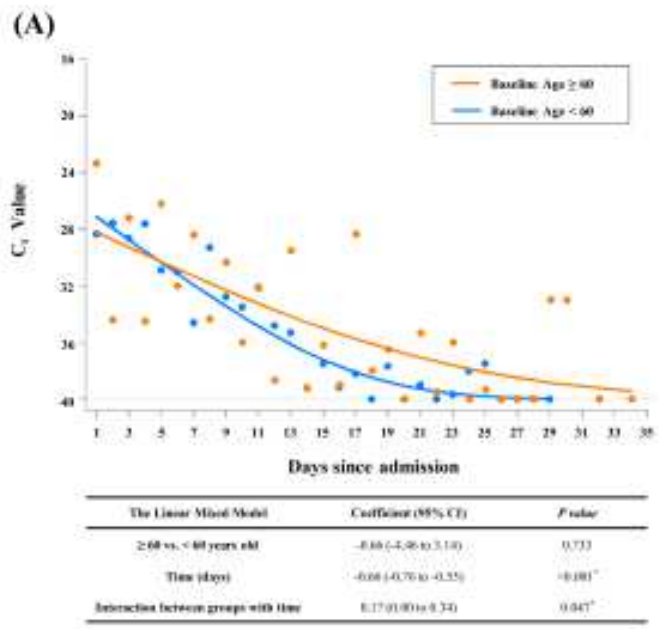

(B)

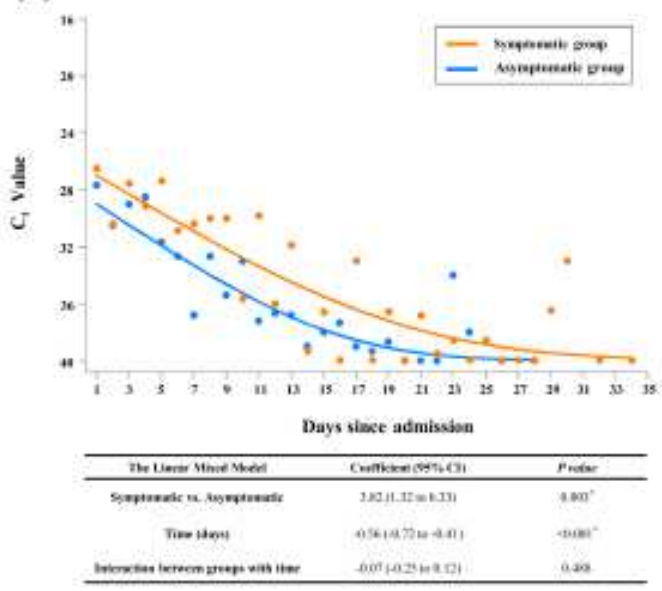

(C)

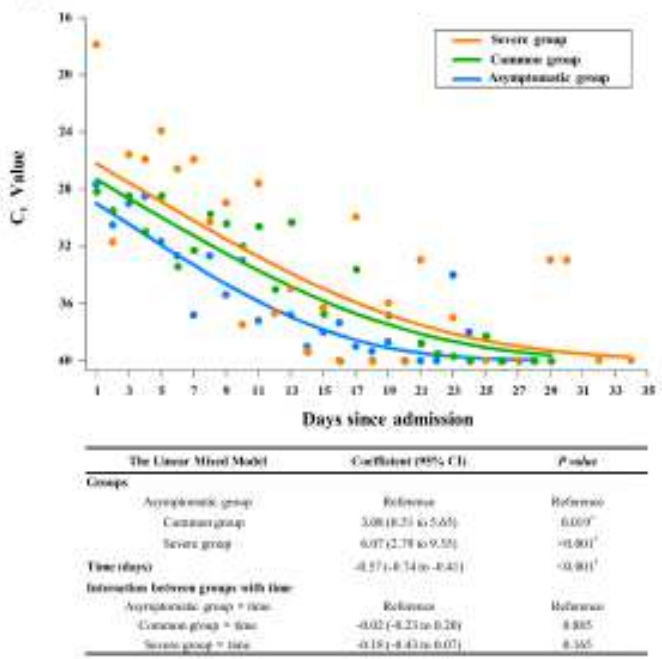

Figure 2

2019-nCov RNA shedding among different clinical groups. Linear mixed models were used to compare the between-group variance, baseline Age (A), Asymptomatic and symptomatic group (B), the common, severe and asymptomatic group (C). 


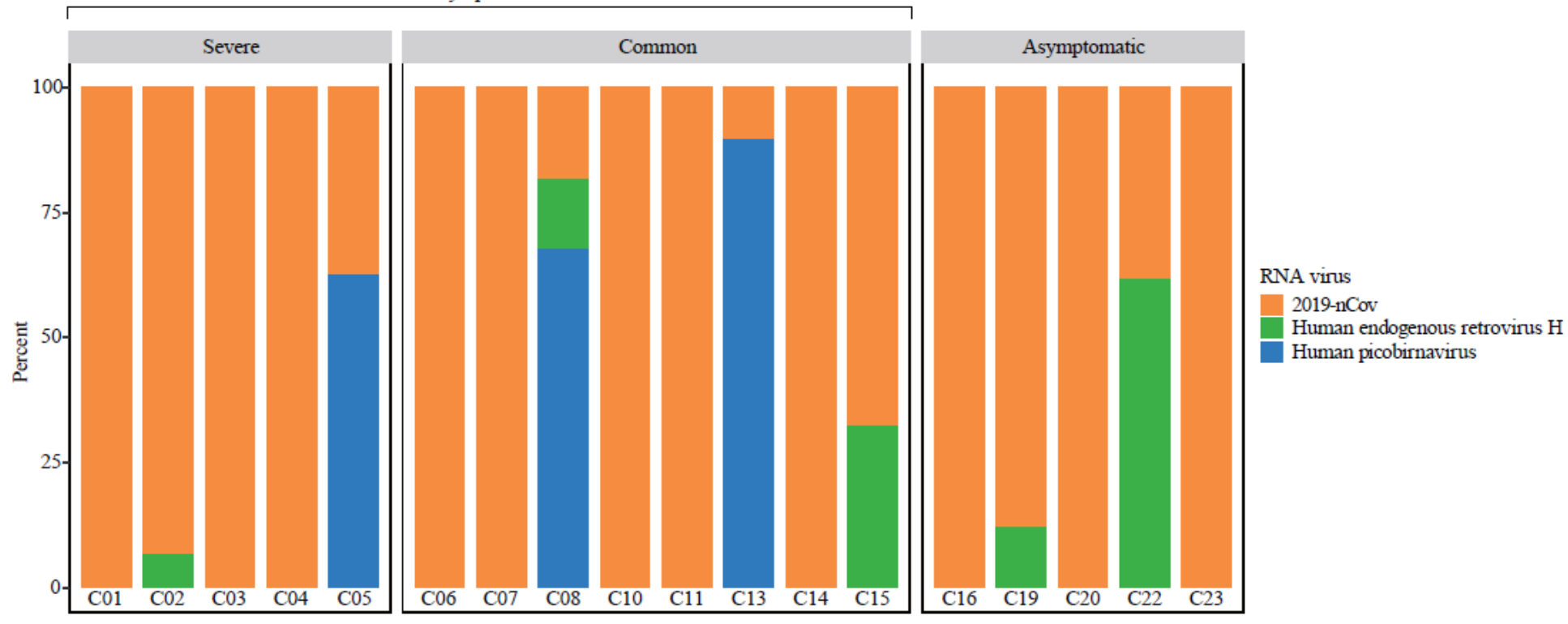

Figure 3

The proportion of three RNA virus through RNA virome sequencing.

\section{Supplementary Files}

This is a list of supplementary files associated with this preprint. Click to download.

- Additionalfile1.docx 